\title{
Duloxetine, a Selective Noradrenaline Reuptake Inhibitor, Increased Plasma Levels of 3-Methoxy-4-hydroxyphenylglycol but Not Homovanillic Acid in Patients with Major Depressive Disorder
}

\author{
Kiyokazu Atake, Reiji Yoshimura, Hikaru Hori, Asuka Katsuki, Atsuko Ikenouchi-Sugita, \\ Wakako Umene-Nakano, Jun Nakamura \\ Department of Psychiatry, University of Occupational and Environmental Health, Kitakyushu, Japan
}

\begin{abstract}
Objective: We investigated the effects of duloxetine on the plasma levels of catecholamine metabolites and serum brain-derived neurotrophic factor (BDNF) in 64 patients with major depressive disorder (MDD).

Methods: Major depressive episode was diagnosed using the Structured Clinical Interview for the Diagnostic and Statistical Manual of Mental Disorders-fourth edition (DSM-IV) according to the DSM-IV text revision (DSM-IV-TR) criteria. The severity of depression was evaluated using the 17-item Hamilton Rating Scale for Depression (HAMD-17). Blood sampling and clinical evaluation were performed on days 0,28 , and 56.

Results: Duloxetine treatment for 8 weeks significantly increased the plasma 3-methoxy-4-hydroxyphenylglycol (MHPG) levels but not the homovanillic acid (HVA) levels in responders with MDD.

Conclusion: These results imply that noradrenaline plays an important role in alleviating depressive symptoms.
\end{abstract}

KEY WORDS: Duloxetine; 3-Methoxy-4-hydroxyphenylglycol; Homovanillic acid; Major depressive disorder; Brain-derived neurotrophic factor.

\section{INTRODUCTION}

Duloxetine, a serotonin noradrenaline reuptake inhibitor (SNRI), is an effective first-line treatment for patients with major depressive disorder (MDD). Recently, duloxetine has also been considered useful for diabetic painful neuropathy and fibromyalgia. ${ }^{1)}$ Generally, duloxetine is safe and well tolerated, although nausea, headache, dry mouth, insomnia, general fatigue, constipation, diarrhea, dizziness, sweating, sexual dysfunction, and appetite loss have been reported in patients with MDD. ${ }^{1)}$ Previously, we reported that milnacipran, another SNRI, increased the plasma levels of 3-methoxy-4-hydroxyphenylglycol (MHPG), a major metabolite of noradrenalin, in patients with MDD. ${ }^{2)}$ Duloxetine potently inhibits both serotonin and noradrenaline transporters.

Received: April 9, 2013 / Revised: June 13, 2013

Accepted: August 6, 2013

Address for correspondence: Reiji Yoshimura, MD, PhD Department of Psychiatry, University of Occupational and Environmental Health, 1-1 Iseigaoka, Yahatanishi-ku, Kitakyushu 8078555, Japan

Tel: +81-936917253, Fax: +81-6924894

E-mail: yoshi621@med.uoeh-u.ac.jp
Therefore, we hypothesized that duloxetine also increases plasma MHPG levels in patients with MDD.

Brain-derived neurotrophic factor (BDNF) plays an important role in the pathogenesis of MDD. We also demonstrated that milnacipran significantly increased serum BDNF levels in patients with MDD compared with healthy subjects. ${ }^{2,3)}$ However, three meta-analyses of studies of blood BDNF levels demonstrated that the serum or plasma BDNF in patients with MDD are lower than are those in healthy contols. ${ }^{4-6)}$ These findings imply that blood BDNF level is a biomarker for MDD. We hypothesized that duloxetine increases serum BDNF levels.

To confirm this hypothesis, this study investigated the effects of duloxetine on the plasma catecholamine metabolite and serum BDNF levels in depressed patients. To our knowledge, this is the first such study.

\section{METHODS}

\section{Subjects}

Sixty-four patients were recruited. Major depressive episode was diagnosed using the structured clinical interview for the Diagnostic and Statistical Manual of Mental

(c) This is an Open-Access article distributed under the terms of the Creative Commons Attribution Non-Commercial License (http://creativecommons.org/licenses/by-nc/3.0) which permits unrestricted non-commercial use, distribution, and reproduction in any medium, provided the original work is properly cited. 
Disorders-fourth edition (DSM-IV) according to DSM-IV text revision (DSM-IV-TR) criteria. The severity of depression was evaluated using the 17-item Hamilton Rating Scale for Depression (HAMD-17). In terms of the somatic symptoms of depression, we included items \#10 (anxiety-psychic), \#11 (anxiety-somatic), \#12 (somatic symptoms-gastrointestinal), \#13 (somatic symptomsgeneral), and \#15 (hypochondriasis) of the HAMD-17. Exclusion criteria were a history of neurological or other physical diseases; comorbidity with other disorders, bipolar disorder, or Axis II personality disorders and mental retardation. No patient received antidepressants or benzodiazepines for at least 1 week before starting the study. We defined patients whose HAMD-17 scores decreased by $50 \%$ or more as responders. Demographic data for duloxetine responders and non-responders are shown in Table 1. Blood sampling and clinical evaluations were performed on days 0,28 (4 weeks), and 56 ( 8 weeks). Of the 64 patients, 45 patients $(70 \%)$ finished the study. The reasons for dropping out are presented in Table 2. Written informed consent was obtained via forms approved by the ethics committee of the University of Occupational and Environmental Health (230737).

Table 1. Demographic data of responder and nonresponder to duloxetine

\begin{tabular}{lccc}
\hline & Responder & Non-responder & $p$ value \\
\hline Sample size (n) & 30 & 15 & \\
Age (year) & $49.2 \pm 15.2$ & $53.3 \pm 15.7$ & 0.348 \\
Sex (M/F) & $16 / 14$ & $7 / 8$ & 0.673 \\
Max dose (mg/day) & $47.3 \pm 13.1$ & $53.3 \pm 9.8$ & 0.137 \\
Base HAMD-17 & $21.3 \pm 4.5$ & $21.1 \pm 5.8$ & 0.681 \\
\hline
\end{tabular}

Values are presented as number only or mean \pm standard deviation. M, male; F, female; HAMD-17, the 17-item Hamilton Rating Scale for Depression.

Table 2. The reason for drop out

\begin{tabular}{lc}
\hline \multicolumn{1}{c}{ Reason } & Number (\%) \\
\hline Drop out & $19(29.69)$ \\
Against the protocol & $8(12.50)$ \\
Changing hospital & $6(9.38)$ \\
Adverse event & $5(7.81)$ \\
Irritation & $2(3.13)$ \\
Nausea & $1(1.56)$ \\
Feel flushed & $1(1.56)$ \\
Auditory hallucination & $1(1.56)$ \\
Total & $64(100.00)$ \\
\hline
\end{tabular}

\section{Methods}

Plasma catecholamine metabolites and serum BDNF assays

The plasma homovanillic acid (HVA) and MHPG concentrations were analyzed using high-performance liquid chromatography with electrochemical detection (HPLCECD) according to our reported method, ${ }^{7)}$ and serum BDNF was measured using a BDNF Emax Immunoassay kit (Promega, Madison, WI, USA) according to the manufacturer's instructions.

\section{Statical analyses}

The Bonferroni method for a repeated-measures analysis of variance (ANOVA) was used for post hoc analysis of comparisons among three groups. Pearson's correlation coefficient was used to compare pairs of groups. The level of significance for all analyses was set at $p<0.05$.

\section{RESULTS}

Thirty of the $45(67 \%)$ patients responded to 8 weeks of duloxetine treatment. Duloxetine treatment for 8 weeks significantly increased the plasma MHPG levels $(p=$ 0.0062 ) in responders but did not change the plasma MHPG levels in non-responders (Fig. 1). Duloxetine did not change the plasma HVA levels in either responders $(p=1.000)$ or non-responders $(p=0.2974)$ (Fig. 2$)$. No significant correlation was observed between changes in the total HAMD-17 scores and changes in plasma MHPG or HVA levels. Additionally, no correlation was found between changes in plasma MHPG or HVA levels and those

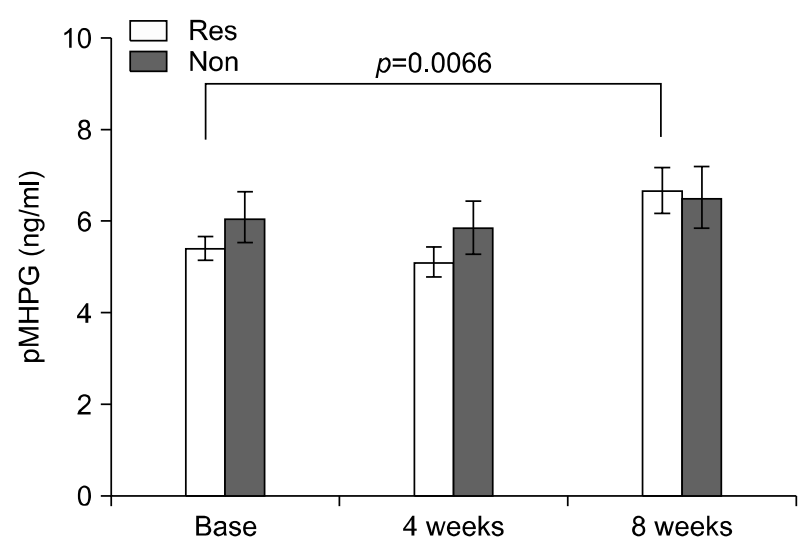

Fig. 1. Changes in plasma 3-methoxy-4-hydroxyphenylglycol (PMHPG) during duloxetine treatment (mean \pm standard deviation).

Res, responders to duloxetine; Non, nonresponders to duloxetine. 


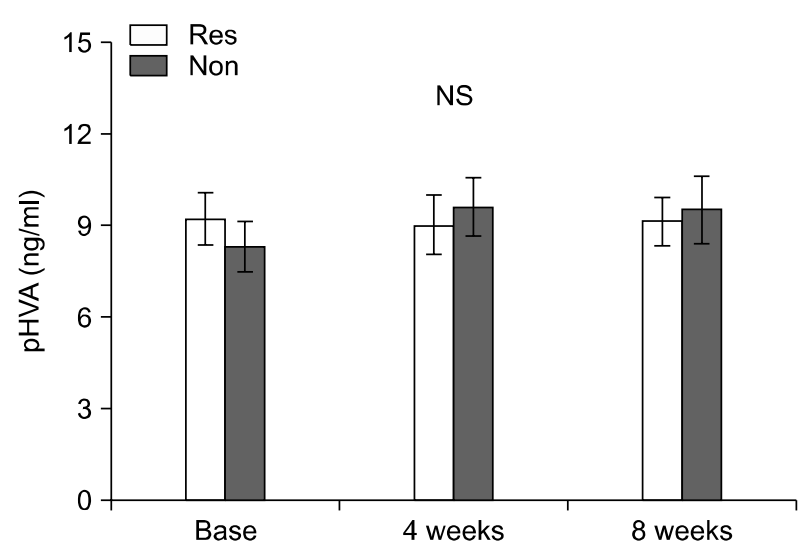

Fig. 2. Changes in plasma homovanillic acid (pHVA) during duloxetine treatment (mean \pm standard deviation).

NS, not significant; Res, responders to duloxetine; Non, nonresponders to duloxetine.

in the subitem scores on the HAMD-17.

Duloxetine treatment for 8 weeks did not significantly increase serum BDNF levels in either responders $(p=$ $0.8055)$ or non-responders $(p=1.000)$. We also analyzed the relationship between the somatic symptoms of depression and the plasma catecholamine metabolite levels in duloxetine responders. No significant positive correlations were found between scores on the somatic symptom scores addressed by the HAMD-17 and baseline levels of plasma MHPG $(r=0.021, p=0.7130)$ or HVA $(r=0.1661$, $p=0.2754)$. An increase in BDNF was not also observed even in responders to duloxetine ( $p=0.3073)$ (Fig. 3).

\section{DISCUSSION}

This is the first report that duloxetine increases plasma MHPG levels in MDD responders. The increase plasma MHPG levels is consistent with the fact that duloxetine effectively inhibits the noradrenaline transporter (NAT). As MDD is a heterogeneous disorder, the levels of plasma MHPG and HVA vary widely in patients ${ }^{2)}$ and are higher in depressed patients than in normal controls; on the other hand, they were lower in depressed patients than in normal controls. Therefore, it is possible that duloxetine acts on noradrenergic neurons in patients with MDD. Noradrenaline is associated primarily with attention, activity, concentration, and pain. Duloxetine might alter the symptoms of MDD by enhancing noradrenaline neurons. Nevertheless, no correlation was observed between changes in plasma MHPG levels and changes in the subitem scores on the HAMD-17. Furthermore, duloxetine did not change plasma HVA levels in the patient group. As there are few dopamine transporters (DATs) in the prefrontal

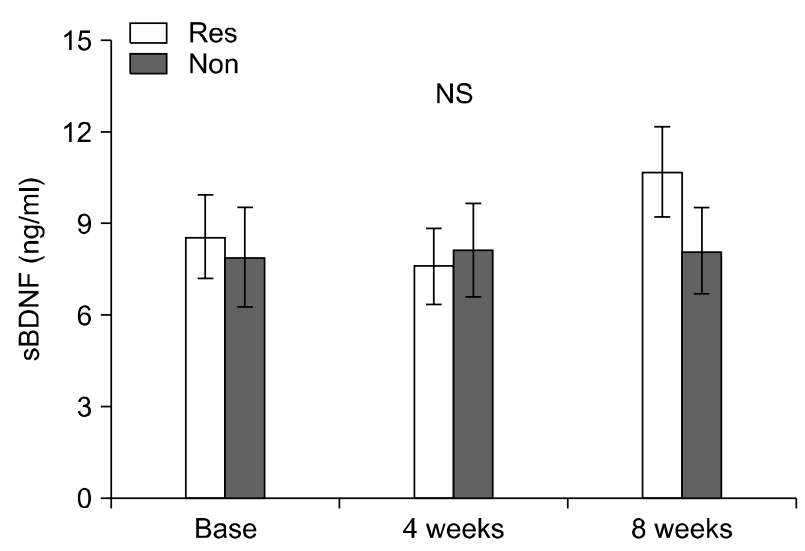

Fig. 3. Changes in serum brain-derived neurotrophic factor (sBDNF) during duloxetine treatment.

NS, not significant; Res, responders to duloxetine; Non, nonresponders to duloxetine.

cortex, dopamine reuptake occurs through the NAT. Therefore, it is plausible that treatment with duloxetine for 8 weeks increases plasma HVA. The fact that duloxetine does not directly inhibit DATs might be the reason for the lack of an observed increase in plasma HVA levels. By contrast, Chalon et al. ${ }^{8)}$ reported that 5-6 days of duloxetine significantly decreased urinary MHPG levels in healthy volunteers. The discrepancy between the studies could be due to differences in sampling methods or study periods. Indeed, the plasma MHPG levels of patients with MDD who responded to milnacipran were significantly lower than were those of healthy subjects. ${ }^{2}$ )

Another important finding of our study was that duloxetine treatment for 8 weeks did not increase serum BDNF levels in responders or non-responders with MDD. All of the subjects in our previous milnacipran study were drug-naïve. By contrast, most of the current patients had been given several antidepressants before staring duloxetine. Although we stopped these drugs for 1 week, this period might have been too short to rule out the effects of the other antidepressants on serum BDNF. Therefore, it is possible that serum BDNF levels were influenced by medications that were received before duloxetine was started.

There are several limitations to this study. First, it was an open-label trial rather than a double-blind, fixed-dose study. Second, the sample size was small, and the drop-out rate was high. Third, there was no healthy control group, and the follow-up period was short. Fourth, plasma MHPG and HVA levels are thought to reflect only $30-50 \%$ and $10-20 \%$ of the dynamics of the brain, respectively. ${ }^{3)}$ Finally, the lack of data on the drop-outs might have had an enormous influence on the results. As most of those patients could be considered non-responders, our results 
might have been more robust than if they had been included. Further research considering these problems should be performed to confirm our preliminary results. In conclusion, treatment with duloxetine for 8 weeks increased plasma MHPG levels in responders but not nonresponders with MDD, which suggests that enhancing noradrenaline neurons plays an important role in alleviating depressive symptoms.

\section{Acknowledgments}

Professor Nakamura has received grant support from Astellas Pharma, Janssen Pharmaceutical, Eli Lilly, Glaxo Smith Kline, Pfizer, Daimppon Sumitomo Pharma, Otsuka Phamaceutical, and Chugai Pharmaceutical. The other authors report no financial relationships with commercial interest.

We gratefully appreciate Ms. Kazuko Shimizu for collecting samples and technical supports in the present study.

\section{REFERENCES}

1. Pergolizzi JV Jr, Raffa RB, Taylor R Jr, Rodriguez G, Nalamachu S, Langley P. A review of duloxetine $60 \mathrm{mg}$ once-daily dosing for the management of diabetic peripheral neuropathic pain, fibromyalgia, and chronic musculoskeletal pain due to chronic osteoarthritis pain and low back pain. Pain Pract 2013;13:239-252.
2. Shinkai K, Yoshimura R, Ueda N, Okamoto K, Nakamura J. Associations between baseline plasma MHPG (3methoxy-4-hydroxyphenylglycol) levels and clinical responses with respect to milnacipran versus paroxetine treatment. J Clin Psychopharmacol 2004;24:11-17.

3. Yoshimura R, Mitoma M, Sugita A, Hori H, Okamoto T, Umene $\mathrm{W}$, et al. Effects of paroxetine or milnacipran on serum brain-derived neurotrophic factor in depressed patients. Prog Neuropsychopharmacol Biol Psychiatry 2007; 31:1034-1037.

4. Sen S, Duman R, Sanacora G. Serum brain-derived neurotrophic factor, depression, and antidepressant medications: meta-analyses and implications. Biol Psychiatry 2008; 64:527-532.

5. Bocchio-Chiavetto L, Bagnardi V, Zanardini R, Molteni R, Nielsen MG, Placentino A, et al. Serum and plasma BDNF levels in major depression: a replication study and metaanalyses. World J Biol Psychiatry 2010;11:763-773.

6. Fernandes BS, Gama CS, Ceresér KM, Yatham LN, Fries $\mathrm{GR}$, Colpo G, et al. Brain-derived neurotrophic factor as a state-marker of mood episodes in bipolar disorders: a systematic review and meta-regression analysis. J Psychiatr Res 2011;45:995-1004.

7. Katsuki A, Yoshimura R, Kishi T, Hori H, Umene-Nakano $\mathrm{W}$, Ikenouchi-Sugita A, et al. Serum levels of brain-derived neurotrophic factor (BDNF), BDNF gene Val66Met polymorphism, or plasma catecholamine metabolites, and response to mirtazapine in Japanese patients with major depressive disorder (MDD). CNS Spectr 2012;17:155-163.

8. Chalon SA, Granier LA, Vandenhende FR, Bieck PR, Bymaster FP, Joliat MJ, et al. Duloxetine increases serotonin and norepinephrine availability in healthy subjects: a double-blind, controlled study. Neuropsychopharmacology 2003; $28: 1685-1693$ 